\title{
Famitinib exerted powerful antitumor activity in human gastric cancer cells and xenografts
}

\author{
SAI GE ${ }^{1 *}$, QIYUE ZHANG ${ }^{1 *}$, QIONG HE ${ }^{1}$, JIANLING ZOU ${ }^{1}$, XIJUAN LIU $^{2}$,

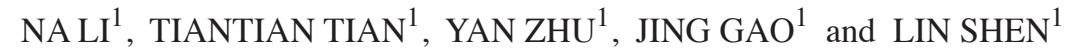 \\ ${ }^{1}$ Department of Gastrointestinal Oncology, ${ }^{2}$ Central Laboratory, Key Laboratory of Carcinogenesis and Translational \\ Research (Ministry of Education), Peking University Cancer Hospital and Institute, Beijing 100142, P.R. China
}

Received April 13, 2015; Accepted June 29, 2016

DOI: $10.3892 / 01.2016 .4909$

\begin{abstract}
Famitinib (SHR1020), a novel multi-targeted tyrosine kinase inhibitor, has antitumor activity against several solid tumors via targeting vascular endothelial growth factor receptor 2, c-Kit and platelet-derived growth factor receptor $\beta$. The present study investigated famitinib's activity against human gastric cancer cells in vitro and in vivo. Cell viability and apoptosis were measured, and cell cycle analysis was performed following famitinib treatment using 3-(4,5-dimethylthiazol -2-yl)-5-(3-carboxymethoxyphenyl)-2-(4-sulfophenyl )-2H-tetrazolium assay, flow cytometry, terminal deoxynucleotidyl transferase dUTP nick end labeling assay and western blotting. Subsequently, cluster of differentiation 34 staining was used to evaluate microvessel density. BGC-823-derived xenografts in nude mice were established to assess drug efficacy in vivo. Famitinib inhibited cell proliferation by inducing cell cycle arrest at the G2/M phase and caused cell apoptosis in a dose-dependent manner in gastric cancer cell lines. In BGC-823 xenograft models, famitinib significantly slowed tumor growth in vivo via inhibition of angiogenesis. Compared with other chemotherapeutics such as 5-fluorouracil, cisplatin or paclitaxel alone, famitinib exhibited the greatest tumor suppression effect ( $>85 \%$ inhibition). The present study demonstrated for the first time that famitinib has efficacy against human gastric cancer in vitro and in vivo, which may lay the foundations for future clinical trials.
\end{abstract}

Correspondence to: Professor Lin Shen or Professor Jing Gao, Department of Gastrointestinal Oncology, Peking University Cancer Hospital and Institute, Fu-Cheng Road 52, Hai-Dian, Beijing 100142, P.R. China

E-mail: lin100@medmail.com.cn

E-mail: gaojing_pumc@163.com

${ }^{*}$ Contributed equally

Key words: famitinib, tyrosine kinase inhibitor, gastric cancer, antitumor activity

\section{Introduction}

In China, $>60 \%$ of gastric cancer patients are initially diagnosed with locally advanced (or metastatic) gastric cancer (AGC) (1), and fluorouracil-based combination chemotherapy is considered a first-line treatment (2). However, clinical outcomes for AGC patients are unsatisfactory, with treatment offering modest responses and poor prognoses (3). Trastuzumab added to this therapy has been documented to prolong the survival of human epidermal growth factor receptor 2 (HER2)-positive AGC patients, but HER2 expression is only reported to occur in $15-20 \%$ of all gastric cancers (4). Thus, novel gastric cancer therapies are required.

In the past, tyrosine kinase inhibitors (TKIs), including imatinib (5), gefitinib (6) and erlotinib (7), have been successfully used to treat other tumors (8), but these drugs have not been used for gastric cancer, despite animal data suggesting their potential efficacy (9). Famitinib (SHR1020) is a novel multi-targeted receptor TKI that targets vascular endothelial growth factor receptor 2 (VEGFR2), c-Kit and platelet-derived

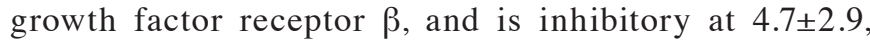
$2.3 \pm 2.6$ and $6.6 \pm 1.1 \mathrm{nM}$, respectively (10). Famitinib is a structural analog of sunitinib with improved cell inhibitory activity (Xie et al, unpublished data). Due to its anti-angiogenic effect, famitinib was effective against metastatic renal cell carcinoma and metastatic breast cancer (11-14). Clinical trials of famitinib against other solid tumors such as advanced colorectal cancer and gastroenteropancreatic neuroendocrine tumors are underway (https://clinicaltrials.gov/). The present study demonstrates the potential antitumor activity of famitinib against human gastric cancer cells in vitro and in vivo.

\section{Materials and methods}

Drugs. Famitinib (SHR1020) was a gift from Jiangsu Hengrui Medicine Co., Ltd. (Lianyungang, China), and its appearance was a yellow powder. The drug was stored at $4^{\circ} \mathrm{C}$ in the dark. For in vitro studies, famitinib was dissolved in dimethylsulfoxide at $20 \mathrm{mmol} / \mathrm{l}$ and stored at $-20^{\circ} \mathrm{C}$ until use. For in vivo animal experiments, famitinib was formulated in physiological saline as a homogeneous suspension $(10 \mathrm{mg} / \mathrm{ml})$ and stored at $4{ }^{\circ} \mathrm{C}$ protected from light. Injectable 5 -florouracil $(5-\mathrm{FU}$, $250 \mathrm{mg} / 10 \mathrm{ml}$ ) was purchased from Tianjin Jinyao Amino 
Acid Co., Ltd. (Tianjin, China). Cisplatin lyophilized powder (DDP, $10 \mathrm{mg}$ ) was purchased from Qilu Pharmaceutical Co., Ltd. (Jinan, China) and was formulated in physiological saline. Paclitaxel (PTX, $30 \mathrm{mg} / 5 \mathrm{ml}$ ) injection was purchased from Hainan Haiyao Co., Ltd. (Hainan, China) and was formulated in physiological saline.

Cell lines and cell culture. Human gastric cancer cells BGC-823 and MGC-803 were provided by Professor Youyong Lv (Peking University Cancer Hospital and Institute, Beijing, China). Both cell lines were cultured in RPMI-1640 medium (Gibco; Thermo Fisher Scientific, Inc., Waltham, MA, USA) supplemented with $10 \%$ fetal bovine serum (Gibco; Thermo Fisher Scientific, Inc.) and incubated in a humidified $37^{\circ} \mathrm{C}$ incubator with $5 \% \mathrm{CO}_{2}$.

3-(4,5-dimethylthiazol -2-yl)-5-(3-carboxymethoxyphenyl) -2-(4-sulfophenyl)-2H-tetrazolium (MTS) cell proliferation assay. Both cell lines were seeded at $\sim 3,000-5,000$ cells/well in a 96-well plate and incubated overnight in complete medium, followed by treatment with different concentrations of famitinib for 24, 48 and $72 \mathrm{~h}$. Cell viability was measured using MTS tetrazolium substrate (CellTiter $96^{\circledR}$ AQueous One Solution Cell Proliferation Assay; Promega Corporation, Madison, WI, USA) according to the manufacturer's protocol. The absorbance was measured at $490 \mathrm{~nm}$ using a spectrophotometer. All experiments were repeated three times with at least triplicates for each concentration.

Cell cycle analysis. Cell were treated with famitinib for $48 \mathrm{~h}$, followed by harvesting and fixing in $70 \%$ cold ethanol for $\geq 12 \mathrm{~h}$ at $4^{\circ} \mathrm{C}$. Cells were stained with $50 \mu \mathrm{g} / \mathrm{ml}$ propidium iodide (BD Biosciences, Franklin Lakes, NJ, USA) at room temperature for $30 \mathrm{~min}$ in the dark, and the cell cycle was analyzed using a FACSAria or a FACSCalibur (BD Biosciences). Data were analyzed by ModFit 3.0 software (BD Biosciences). All experiments were performed in triplicate.

Terminal deoxynucleotidyl transferase dUTP nick end labeling (TUNEL) assay. Cell apoptosis was measured via TUNEL assay (catalog no., C1086; Beyotime Institute of Biotechnology, Haimen, China) according to the manufacturer's protocol. Upon treatment of the cells with famitinib for $48 \mathrm{~h}$, cells were washed with phosphate-buffered saline (PBS) and fixed with $4 \%$ paraformaldehyde for $10 \mathrm{~min}$ at room temperature. Cells were then stained with the corresponding reagents provided in the TUNEL assay kit. Upon overlaying the coverslips, slides were imaged under fluorescence microscopy (TCS SP5; Leica Microsystems GmbH, Wetzlar, Germany). Positive cells exhibited green fluorescence and were counted from three random microscopic fields.

Western blotting. Total proteins prior and subsequent to famitinib treatment were extracted from BGC-823 and MGC-803 cell pellets using CytoBuster Protein Extraction Reagent (Merck Millipore, Darmstadt, Germany). Proteins were quantified with a Pierce BCA Protein Assay kit (Thermo Fisher Scientific, Inc.), and $\sim 20 \mu$ of protein was separated on $15 \%$ sodium dodecyl sulfate-polyacrylamide gel electrophoresis, and proteins were then transferred to a nitrocellulose membrane (GE Healthcare Life Sciences, Chalfont, UK), which was subsequently incubated with anti-cyclin B1 (dilution, 1:1,000; catalog no., AJ1208a; Abgent Inc., San Diego, CA, USA), rabbit polyclonal anti-B-cell lymphoma 2 (BCL2; dilution, 1:1,000; catalog no., 2872; Cell Signaling Technology, Inc., Danvers, MA, USA) and mouse monoclonal anti- $\beta$-actin (dilution, 1:3,000; catalog no., A5441; Sigma-Aldrich, St. Louis, MO, USA) antibodies at $4^{\circ} \mathrm{C}$ overnight. Secondary anti-rabbit and anti-mouse horseradish peroxidase-conjugated IgG antibodies (dilution, 1:3,000; catalog nos., 7074 and 7076, respectively; Cell Signaling Technology, Inc.) were applied and allowed to incubate at room temperature for $1 \mathrm{~h}$. Proteins were visualized with ECL Plus Western Blotting Detection Reagent (GE Healthcare Life Sciences).

In vivo xenograft model experiments. BGC-823 cells were suspended in PBS $\left(1 \times 10^{7}\right.$ cells $\left./ \mathrm{ml}\right)$, and $100 \mu \mathrm{l}$ of the cell suspension was subcutaneously injected into the right axillary area of $18-20-\mathrm{g}$ female BALB/c athymic $n u / n u$ mice ( $n=40$; age, 6-8 weeks; Vital River Laboratories Co., Ltd., Beijing, China). The temperature of the housing conditions was maintained at $23-25^{\circ} \mathrm{C}$ with a humidity of $50-60 \%$ and a 10/14 h light/dark cycle. Food and water were changed 3 times a week. When the tumor volume reached $\sim 100 \mathrm{~mm}^{3}$, mice were randomized into treatment groups. Tumors and animal weights were measured twice weekly, and tumor volume was calculated using the following formula: $\mathrm{V}=\mathrm{LxW}^{2} \mathrm{x} 1 / 2$ (where $\mathrm{V}$ represents tumor volume, $\mathrm{L}$ is the length of the tumor and $\mathrm{W}$ is the width of the tumor).

To measure famitinib, three groups were randomized ( $\mathrm{n}=5 \mathrm{mice} / \mathrm{group}$ ) as follows: Control group (gavage, physiological saline, once daily for 3 weeks); low-dose famitinib group (gavage, $50 \mathrm{mg} / \mathrm{kg}$, once daily for 3 weeks); and high-dose famitinib group (gavage, $100 \mathrm{mg} / \mathrm{kg}$, once for 3 weeks). A dose of $50 \mathrm{mg} / \mathrm{kg}$ was used for the following experiments.

To compare famitinib with other drugs, animals were randomized ( $\mathrm{n}=5 \mathrm{mice} / \mathrm{group}$ ) as follows: Control group (gavage, physiological saline, once daily for 3 weeks); famitinib group (gavage, $50 \mathrm{mg} / \mathrm{kg}$, once daily for 3 weeks); $5-\mathrm{FU}$ group $[10 \mathrm{mg} / \mathrm{kg}$, intraperitoneal (ip), once every 2 days for 3 weeks]; DDP group ( $3 \mathrm{mg} / \mathrm{kg}$, ip, once weekly for 3 weeks); and PTX group (10 mg/kg, ip, once a week for 3 weeks). Then, tumors and weight were quantified. All animal experiments were approved by the Institutional Ethics and Animal Care Committee and performed in accordance with the animal experimental guidelines of Peking University Cancer Hospital (Beijing, China).

Hematoxylin and eosin $(H \& E)$ and immunohistochemical staining. Once the mice were sacrificed, xenografts were isolated, and formalin-fixed, paraffin-embedded (FFPE) tissue blocks were obtained. FFPE tumor sections $(4-\mu \mathrm{m}$ thick) were deparaffinized in xylene and hydrated in graded alcohol. Then, tumor sections were either stained using a H\&E staining kit (catalog no., C0105; Beyotime Institute of Biotechnology), according to the manufacturer's protocol, or incubated with rabbit polyclonal anti-cluster of differentiation (CD)34 antibody (dilution, 1:2,500; catalog no., ab81289; Abcam, Cambridge, UK) upon antigen retrieval and endogenous peroxidase treatment. Signals were visualized using an 

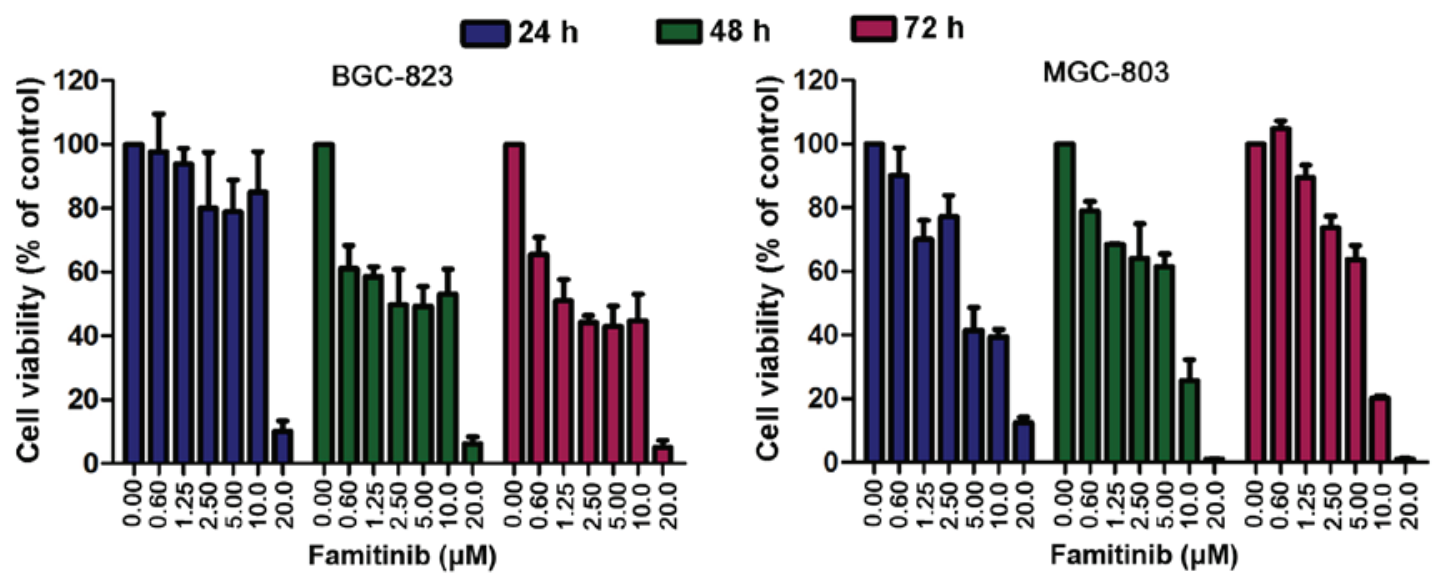

Figure 1. Inhibition of cell growth mediated by famitinib in BGC-823 and MGC-803 cell lines. Cells were exposed to famitinib for 24,48 and $72 \mathrm{~h}$, and cell viability was diminished in a dose-dependent manner.

immunoglobulin G-horseradish peroxidase polymer (Beijing CoWin Biotech Co., Ltd., Beijing, China) and 3,3'-diaminobenzidine substrate. Sections were scored by two independent pathologists as previously described (15).

Statistical analysis. SPSS 18.0 software (SPSS, Inc., Chicago, IL, USA) was used to perform the statistical analysis. One-way analysis of variance was used for the in vivo experiments. $\mathrm{P}<0.05$ was considered to indicate a statistically significant difference.

\section{Results}

Famitinib inhibits gastric cancer cell growth in a dose-dependent manner. BGC-823 and MGC-803 cells were treated with famitinib $(0,0.6,1.25,2.5,5.0,10.0$ and $20.0 \mu \mathrm{M})$ for 24, 48 and $72 \mathrm{~h}$, followed by MTS assay. Famitinib inhibited cell growth in a dose-dependent manner (Fig. 1). The half maximal inhibitory concentration $\left(\mathrm{IC}_{50}\right.$ ) values of famitinib in BGC-823 and MGC-803 cells were 3.6 and $3.1 \mu \mathrm{M}$, respectively. Based on these results, the $\mathrm{IC}_{50}$ and $1 / 2 \mathrm{IC}_{50}$ were used for in vitro experiments.

Famitinib induces cell cycle arrest at the G2/M phase. The cell cycle was next analyzed following famitinib treatment, and it was observed that the number of G2/M-phase cells increased in BGC-823 (27.98 vs. 14.25\%) and MGC-803 (58.23 vs. $10.72 \%)$ cells compared with the control $(\mathrm{P}=0.04$ and $\mathrm{P}=0.02$, respectively; Fig. $2 \mathrm{~A}$ and $\mathrm{B}$ ). Cell cycle arrest at the $\mathrm{G} 2 / \mathrm{M}$ phase was confirmed by increased expression of the cell metaphase-specific protein cyclin B1 (Fig. 2C).

Famitinib triggers apoptosis. Cell apoptosis is an important mechanism of cell growth inhibition (16); therefore, apoptosis was measured via TUNEL assay. Fig. 3 indicates that, compared with the control, famitinib increased apoptosis in BGC-823 and MGC-803 cell lines significantly $(\mathrm{P}<0.01)$, and downregulated BCL2.

Famitinib reduces xenograft growth in vivo via inhibition of angiogenesis. To identify the optimal famitinib dose for the in vivo study, two doses were used, 50 and $100 \mathrm{mg} / \mathrm{kg}$. Both doses exerted a similar inhibitory power, but greater toxicity was observed with the highest dose (data not shown).

Mice were sacrificed 21 days after treatment, and tumors were isolated. Famitinib inhibited BGC-823 xenograft growth (tumor volume, 395.2 vs. 2,690.5 mm³, $\mathrm{P}<0.01$; Fig. 4A), and animal weights were similar between groups (21.6 vs. $18.7 \mathrm{~g}$, $\mathrm{P}=0.17$; Fig. 4B).

Famitinib is considered to inhibit angiogenesis; therefore, microvessel density was measured with CD34 staining. Upon famitinib treatment, xenografts had greater tissue necrosis (Fig. 4C) and exhibited significantly weaker CD34 staining than the controls (Fig. 4D). Thus, famitinib inhibits tumor vascularization.

Famitinib has greater tumor inhibitory effect than 5-FU, $D D P$ or $P T X$. In clinical practice, 5-FU, DDP and PTX are the most commonly used chemotherapeutic drugs for gastric cancer. Thus, the activity of famitinib alone was compared with that of these compounds, and it was observed that famitinib exerted better tumor inhibition than 5-FU, DDP and PTX (Fig. 4E and F). The mean tumor volumes of the control, 5-FU, DDP, PTX and famitinib groups were 1,973.0, 1,680.3, 987.3, 1,577.6 and $287.6 \mathrm{~mm}^{3}$, respectively; and the corresponding tumor inhibitory ratios were $0.0,14.9,49.9,20.0$ and $85.4 \%$, respectively.

\section{Discussion}

According to the data from phase I studies of famitinib against advanced solid tumors (11), one third of patients with AGC responded to famitinib and had a stabilized disease without further evaluation. Thus, the present data offer additional evidence for future trials of famitinib against gastric cancer. The current study confirmed that famitinib alone inhibited the growth of BGC-823 and MGC-803 gastric cancer cells in a dose-dependent manner in vitro (Fig. 1). From the in vivo results, it was concluded that the inhibitory effect of famitinib in mice xenografts was greater than that of 5-FU, DDP or PTX alone (Fig. 4E and F). Thus, famitinib has promising antitumor activity against gastric cancer. For 

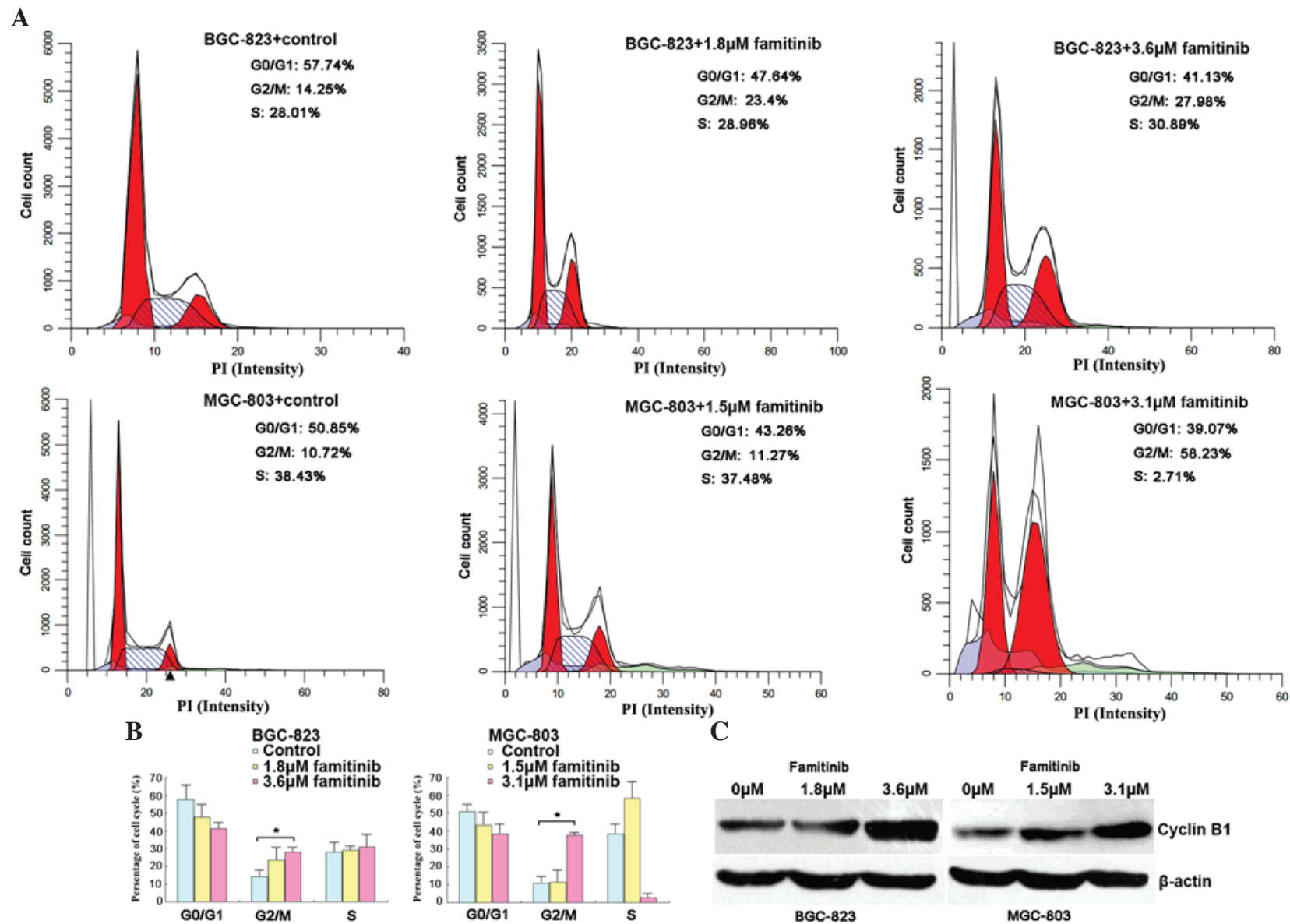

Figure 2. Famitinib induced cell cycle arrest at the G2/M phase. (A and B) Cell cycle was arrested in cell lines BGC-823 and MGC-803 ( $\mathrm{n}=3$; mean \pm SD; ${ }^{*} \mathrm{P}<0.05$ ). (C) With famitinib treatment, cyclin B1 was upregulated in both cell lines according to western blot analysis. SD, standard deviation; PI, propidium iodide.

A
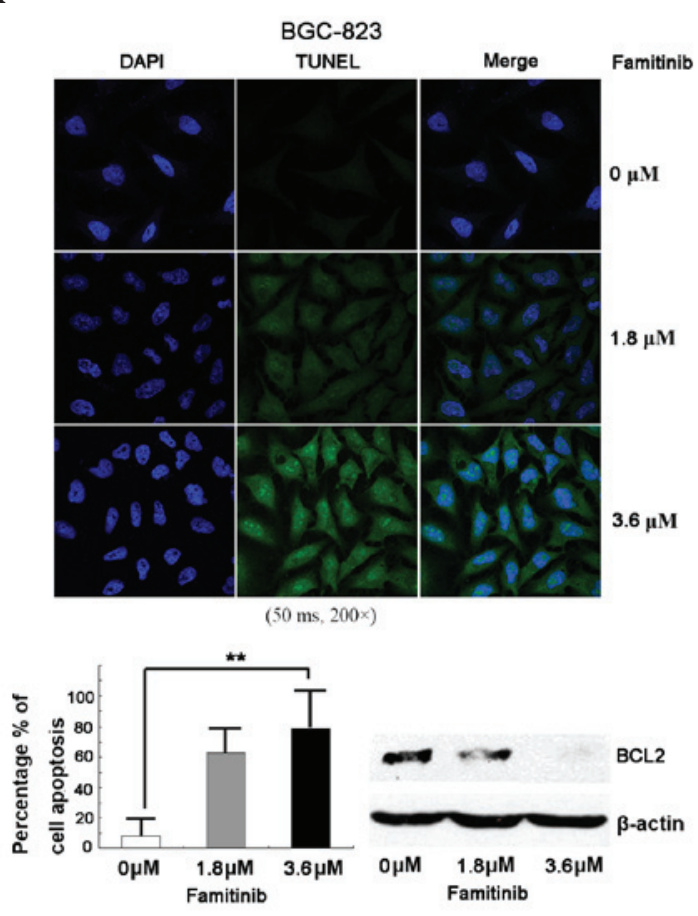

B

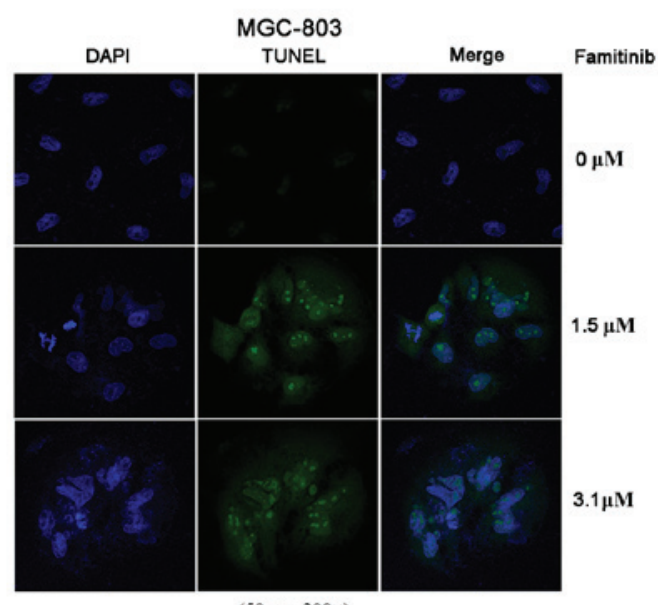

$(50 \mathrm{~ms}, 200 \times)$

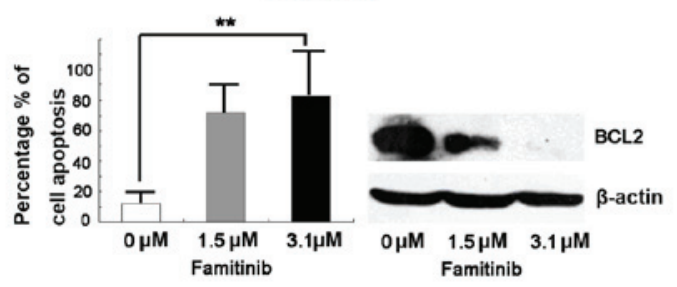

Figure 3. Famitinib triggered cell apoptosis. (A and B) Famitinib induced cell apoptosis in (A) BGC-823 and (B) MGC-803 cells compared with the control $\left(\mathrm{n}=3\right.$, mean \pm standard deviation, $\left.{ }^{* *} \mathrm{P}<0.01\right)$, and downregulated B-cell lymphoma 2 in both cell lines. TUNEL, terminal deoxynucleotidyl transferase dUTP nick end labeling; DAPI, 4',6-diamidino-2-phenylindole; BCL2, B-cell lymphoma 2. 
A

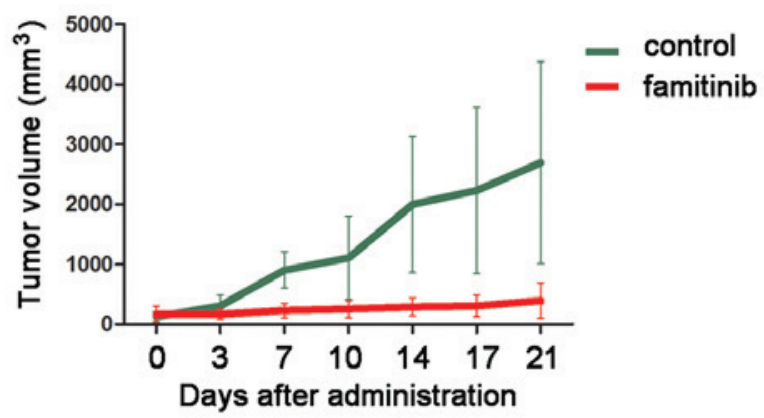

C

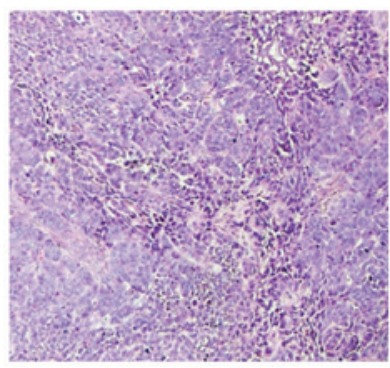

control

E

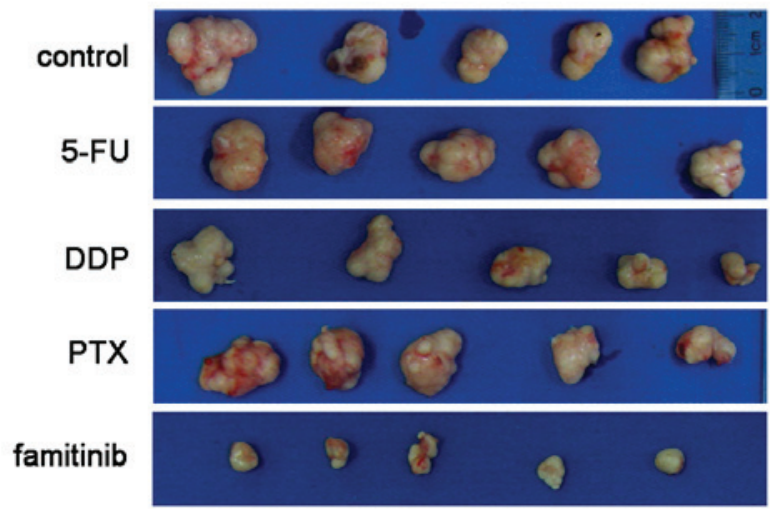

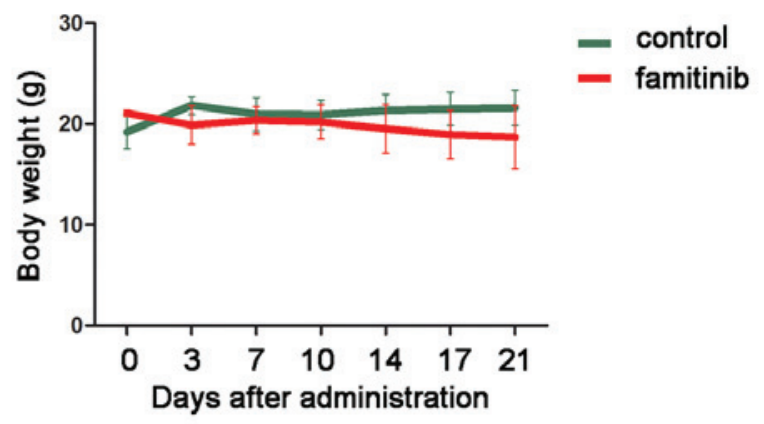

D

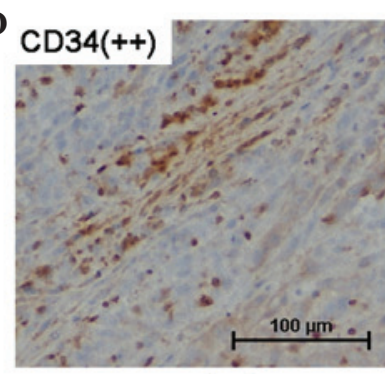

control

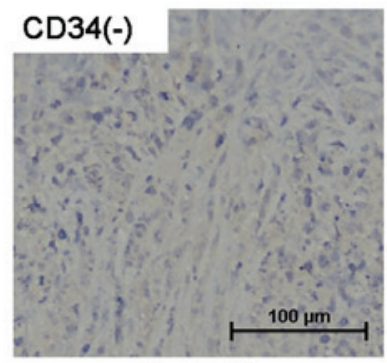

famitinib

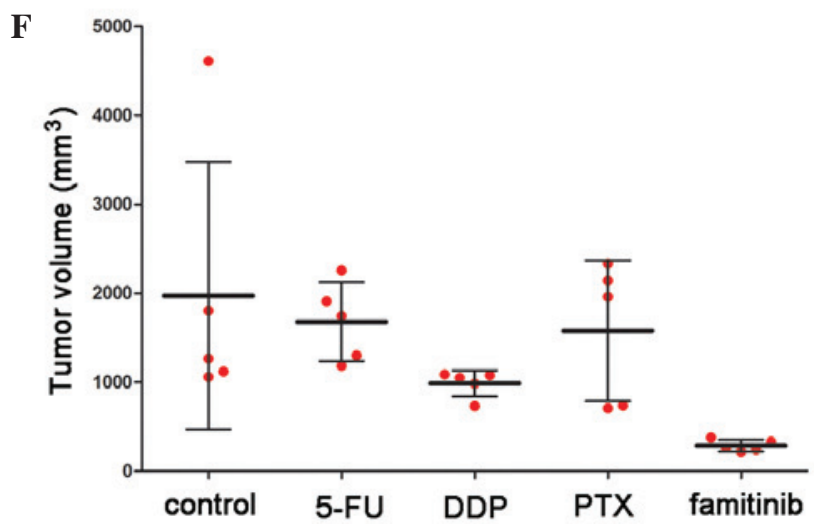

Figure 4. Inhibitory effect of famitinib alone compared with 5-fluorouracil, cisplatin or paclitaxel alone on in BGC-823 xenografts. Growth curves of (A) xenografts and (B) animal weight treated with famitinib $(50 \mathrm{mg} / \mathrm{kg}$ ) or control ( $\mathrm{n}=5 \mathrm{mice} / \mathrm{group}$, mean $\pm \mathrm{SD})$. (C) H\&E staining revealed areas of tissue necrosis following famitinib treatment (") (magnification, x200). (D) Tumor vascularization was significantly suppressed by famitinib according to CD34 expression (magnification, x200). (E) Upon treatment, tumor xenografts were isolated and photographed. (F) Tumor volumes from each group were measured $(\mathrm{n}=5$ mice/group, mean $\pm \mathrm{SD})$. CD, cluster of differentiation; 5-FU, 5-fluorouracil; DDP, cisplatin; PTX, paclitaxel; SD, standard deviation.

animal experiments, 5-FU, DDP and PTX alone were dosed at 10,3 and $10 \mathrm{mg} / \mathrm{kg}$, respectively. According to our previous study (15) and the results from the present study (data not shown), although high doses of 5-FU, DDP and PTX (20, 6 and $20 \mathrm{mg} / \mathrm{kg}$, respectively) alone had better antitumor activity than low doses of the same drugs, these were all toxic at higher doses. Furthermore, based on our preliminary results, the antitumor activity of famitinib was greater than high doses of 5-FU, DDP and PTX.

Combination regimens of $\geq 2$ drugs for gastric cancer are commonly used in clinical practice (2). In the present study, famitinib was not used with other drugs due to its impressive inhibitory activity ( $>85 \%)$. Thus, we anticipate that patients who do not have success with traditional treatment may be treated with famitinib for AGC and advanced colorectal cancer (NCT01762293; clinicaltrials.gov/ct2/show/NCT0176229).

The present study revealed that famitinib induced cell cycle arrest at the $\mathrm{G} 2 / \mathrm{M}$ phase, which was validated by the upregulation of cyclin B1 (Fig. 2C). In addition, cell apoptosis was triggered by famitinib, according to the results of TUNEL assay, and this was confirmed by the downregulation of BCL2 (Fig. 3). Anti-angiogenesis was reported to be an important mechanism of famitinib (13), which was confirmed by the present study. Upon famitinib treatment, tumor microvessel density represented by CD34 staining was significantly lower than that exhibited by the controls (Fig. 4D). Famitinib may have other targets, which may be studied in future experiments.

Anti-angiogenic therapy has been used since it was first proposed by Dr Judah Folkman in 1971 (17). Although the frequent failures of anti-angiogenic drugs such as bevacizumab and sorafenib have been documented in the treatment of gastric cancer (18-20), ramucirumab and apatinib, which mainly block VEGFR2, do improve the survival of patients with chemotherapy-refractory AGC compared with placebo (21-23). Similar to ramucirumab and apatinib, famitinib mainly targets VEGFR2, which was not investigated in the present study. However, tumor microvessel density decreased upon famitinib treatment compared with the controls (Fig. 4D). 
In additional studies, we will futher validate the inhibitory effect of famitinib in gastric cancer patient-derived xenografts, as well as the synergistic effects of famitinib combined with common chemotherapeutic drugs. In conclusion, the present study demonstrated that famitinib induces cell cycle arrest at the $\mathrm{G} 2 / \mathrm{M}$ phase and causes cell apoptosis and anti-angiogenesis. The present data can be used as a foundation for future studies to identify drugs to treat gastric cancer.

\section{Acknowledgements}

The present study was supported by the National Natural Science Foundation of China (Beijing, China; grant nos. 81172110, 81472789 and 81301853) and the National High Technology Research and Development Program (Beijing, China; grant no. 2012AA 02A 504). The authors would like to thank LetPub (Woburn, MA, USA) for its linguistic assistance during the preparation of the present manuscript.

\section{References}

1. Ferlay J, Soerjomataram I, Dikshit R, Eser S, Mathers C, Rebelo M, Parkin DM, Forman D and Bray F: Cancer incidence and mortality worldwide: Sources, methods and major patterns in GLOBOCAN 2012. Int J Cancer 136: E359-E386, 2015.

2. Ajani JA, Bentrem DJ, Besh S, D'Amico TA, Das P, Denlinger C, Fakih MG, Fuchs CS, Gerdes H, Glasgow RE, et al; National Comprehensive Cancer Network: Gastric cancer, version 2.2013: featured updates to the NCCN Guidelines. J Natl Compr Canc Netw 11: 531-546, 2013.

3. Shen L, Shan YS, Hu HM, Price TJ, Sirohi B, Yeh KH, Yang YH, Sano T, Yang HK, Zhang X, et al: Management of gastric cancer in Asia: Resource-stratified guidelines. Lancet Oncol 14 e535-e547, 2013.

4. Bang YJ, Van Cutsem E, Feyereislova A, Chung HC, Shen L, Sawaki A, Lordick F, Ohtsu A, Omuro Y, Satoh T, et al: Trastuzumab in combination with chemotherapy versus chemotherapy alone for treatment of HER2-positive advanced gastric or gastro-oesophageal junction cancer (ToGA): A phase 3, open-label, randomised controlled trial. Lancet 376: 687-697, 2010.

5. Druker BJ, Tamura S, Buchdunger E, Ohno S, Segal GM, Fanning S, Zimmermann J and Lydon NB: Effects of a selective inhibitor of the Abl tyrosine kinase on the growth of Bcr-Abl positive cells. Nat Med 2: 561-566, 1996.

6. Barker AJ, Gibson KH, Grundy W, Godfrey AA, Barlow JJ, Healy MP, Woodburn JR, Ashton SE, Curry BJ, Scarlett L, et al: Studies leading to the identification of ZD1839 (IRESSA): An orally active, selective epidermal growth factor receptor tyrosine kinase inhibitor targeted to the treatment of cancer. Bioorg Med Chem Lett 11: 1911-1914, 2001.

7. Perez-Soler R: The role of erlotinib (Tarceva, OSI 774) in the treatment of non-small cell lung cancer. Clin Cancer Res 10: 4238s-4240s, 2004.

8. Rask-Andersen M,Zhang J, Fabbro D and Schiöth HB: Advances in kinase targeting: Current clinical use and clinical trials. Trends Pharmacol Sci 35: 604-620, 2014.
9. Yang W, Raufi A and Klempner SJ: Targeted therapy for gastric cancer: Molecular pathways and ongoing investigations. Biochim Biophys Acta 1846: 232-237, 2014

10. Lou L, Mi Y, Xu Y, Xie C and Zhao H: Preclinical antitumor study of famitinib, an orally available multi-targeted kinase inhibitor of VEGFR/PDGFR/c-Kit in phase I clinical trials. In: Proceedings of AACR 102nd Annual Meeting. Cancer Res, Orlando, FL, pp71, 2011.

11. Zhou A, Zhang W, Chang C, Chen X, Zhong D, Qin Q, Lou D, Jiang $\mathrm{H}$ and Wang J: Phase I study of the safety, pharmacokinetics and antitumor activity of famitinib. Cancer Chemother Pharmacol 72: 1043-1053, 2013.

12. Zhang W, Zhou AP, Qin Q, Chang CX, Jiang HY, Ma JH and Wang JW: Famitinib in metastatic renal cell carcinoma: A single center study. Chin Med J (Engl) 126: 4277-4281, 2013.

13. Xie C, Zhou J, Guo Z, Diao X, Gao Z, Zhong D, Jiang H, Zhang L and Chen X: Metabolism and bioactivation of famitinib, a novel inhibitor of receptor tyrosine kinase, in cancer patients. Br J Pharmacol 168: 1687-1706, 2013

14. Cao J, Zhang J, Wang Z, Wang B, Lv F, Wang L and Hu X: Hypothyroidism as a potential biomarker of efficacy of famitinib, a novel VEGFR-2 inhibitor in metastatic breast cancer. Cancer Chemother Pharmacol 74: 389-398, 2014.

15. He Q, Gao J, Ge S, Wang T, Li Y, Peng Z, Li Y and Shen L: Axitinib alone or in combination with chemotherapeutic drugs exerts potent antitumor activity against human gastric cancer cells in vitro and in vivo. J Cancer Res Clin Oncol 140: 1575-1583, 2014.

16. Shen L, Shan YS, Hu HM, Price TJ, Sirohi B, Yeh KH, Yang YH, Sano T, Yang HK, Zhang X, et al: Management of gastric cancer in Asia: Resource-stratified guidelines. Lancet Oncol 14: e535-e547, 2013.

17. Folkman J: Tumor angiogenesis: Therapeutic implications. N Engl J Med 285: 1182-1186, 1971.

18. Shen L, Li J, Xu J, Pan H, Dai G, Qin S, Wang L, Wang J, Yang Z, Shu Y, et al: Bevacizumab plus capecitabine and cisplatin in Chinese patients with inoperable locally advanced or metastatic gastric or gastroesophageal junction cancer: Randomized, double-blind, phase III study (AVATAR study). Gastric Cancer 18: 168-176, 2015.

19. Ohtsu A, Shah MA, Van Cutsem E, Rha SY, Sawaki A, Park SR, Lim HY, Yamada Y, Wu J, Langer B, et al: Bevacizumab in combination with chemotherapy as first-line therapy in advanced gastric cancer: A randomized, double-blind, placebo-controlled phase III study. J Clin Oncol 29: 3968-3976, 2011.

20. Martin-Richard M, Gallego R, Pericay C, Garcia Foncillas J, Queralt B, Casado E, Barriuso J, Iranzo V, Juez I, Visa L, et al: Multicenter phase II study of oxaliplatin and sorafenib in advanced gastric adenocarcinoma after failure of cisplatin and fluoropyrimidine treatment. A GEMCAD study. Invest New Drugs 31: 1573-1579, 2013.

21. Ilson DH: Angiogenesis in gastric cancer: Hitting the target? Lancet 383: 4-6, 2014.

22. Fuchs CS, Tomasek J, Yong CJ, Dumitru F, Passalacqua R, Goswami C, Safran H, dos Santos LV, Aprile G, Ferry DR, et al: Ramucirumab monotherapy for previously treated advanced gastric or gastro-oesophageal junction adenocarcinoma (REGARD): An international, randomised, multicentre, placebo-controlled, phase III trial. Lancet 383: 31-39, 2014.

23. Li J, Qin S, Xu J, Guo W, Xiong J, Bai Y, Sun G, Yang Y, Wang L, Xu N, et al: Apatinib for chemotherapy-refractory advanced metastatic gastric cancer: Results from a randomized, placebo-controlled, parallel-arm, phase II trial. J Clin Oncol 31: 3219-3225, 2013. 